[2] Filippucci E, da Luz KR, Di Geso L, et al. Interobserver reliability of ultrasonography in the assessment of cartilage damage in rheumatoid arthritis. Ann Rheum Dis. 2010;69:1845-8.

Disclosure of Interests: Edoardo Cipolletta: None declared, Emilio Filippucci: None declared, Andrea Di Matteo: None declared, Marco Di Carlo: None declared, Fausto Salaffi Grant/research support from: Abbvie, Roche, Novartis, BMS, Pfizer, Sanofi, Speakers bureau: Abbvie, Roche, Novartis, Pfizer, Sanofi, BMS, Walter Grassi: None declared

DOI: 10.1136/annrheumdis-2019-eular.7293

\section{AB1132 THE ROLE OF PET/CT IN THE MANAGEMENT OF GIANT CELL ARTERITIS}

Alessandro Croce $^{1},{ }^{1,2}$ Mattia Bellan ${ }^{3,3}$, Roberta Pedrazzoli ${ }^{1}$, Daniele Sola ${ }^{1}$, Erinda Puta ${ }^{4}$, Gian Mauro Sacchetti ${ }^{4}$, Daniele Soddu', Erika Zecca ${ }^{1}$, ${ }_{1,2}$ Mario Pirisi ${ }^{3}$, Pier Paolo Sainaghi ${ }^{1,2} 3,3,{ }^{1}$ A.O.U. Maggiore della carità, Internal Medicine Division, Immunorheumatology Unit, Novara -, Italy, ${ }^{2}$ Università del Piemonte Orientale - UPO, Department of Translational Medicine, Novara, Italy; ${ }^{3} I R C A D$ - Interdisciplinary center of autoimmune diseases, Novara, Italy; ${ }^{4}$ A.O.U. Maggiore della carità, Nuclear Medicine, Novara, Italy

Background: Giant cell arteritis (GCA) is a large vessel vasculitis that affects the aorta and/or its major branches including the superficial temporal artery. Together to cranial symptoms such as headache and visual disturbances, extra cranial manifestations have been widely reported, sometimes as unique clinical presentation. Several imaging modalities are available to evaluate aortic involvement including fluorodeoxyglucose (FDG) positron emission tomography (PET), however there are few reports that analyzed the impact of the findings of this advanced imaging in predicting outcomes of the disease.

Objectives: To assess the utility of PET in disease extension assessment to predict, together to clinical manifestations, the disease evolution.

Methods: We retrospectively reviewed all the clinical records of patients receiving a diagnosis of large vessel vasculitis from $1^{\text {st }}$ January 2010 to $1^{\text {st }}$ January 2016 at a tertiary immunorheumatology clinic of a university Hospital, who underwent a PET. Clinical, laboratory, and imaging data were collected. Non-parametric analysis was performed.

Results: We recruited 19 patients (10 females, 52.6\%). The median age was 74.0 [65.5-76.0]; at the diagnosis, the median erythrocyte sedimentation rate $(E S R)$ was $65.5[49.0-86.0]$, while C-reactive protein (CRP) was 8.5 [5.5-14.0]. 12 patients showed a typical cranial GCA (63.2\%), while 7 $(36.8 \%)$ were diagnosed with extracranial GCA. The two groups were comparable at diagnosis for age, gender, median ESR and CRP. Interestingly, the PET was significative for aortitis not only in the 7 patients with extracranial involvement, but also in $7 / 12$ patients with cranial GCA (58.3\%). Along a median follow-up of 15 months [4.5-26.5], 4 relapses were reported. Notably, all the relapsers were males and showed both aortic and cranial involvement. In a multivariate model, male gender was the only predictor of relapse $(p=0.02)$, while age at onset, clinical subset (cranial vs. extracranial) and steroid dose did not fit the model

Conclusion: The use of PET in GCA is relevant in the assessment of extension of disease since a significative number of patients without cranial symptoms in the end resulted to have large vessel involvement. In addition, PET is useful in identifying patients with cranial involvement that have also aortic inflammation since they seems to have worse prognosis. Disclosure of Interests: None declared

DOI: 10.1136/annrheumdis-2019-eular.7070

\section{AB1133 AUTOMATED ASSESSMENT (AUTOCAPI) OF NAILFOLD CAPILLARY NUMBER VERSUS MANUAL COUNTING IN SYSTEMIC SCLEROSISPATIENTS WITH DIFFERENT CAPILLAROSCOPIC PATTERNS}

Maurizio Cutolo ${ }^{1},{ }^{2,3}$ Amber Vanhaecke ${ }^{4}$, Carmen Pizzorni ${ }^{1},{ }^{2,3}$ Vanessa Smith ${ }^{4}$, Monica Pendolino ${ }^{1}$, Massimo Patanè ${ }^{1}$, Veronica Tomatis ${ }^{1}$, Alberto Sulli $^{1}$.

${ }^{1}$ Research Laboratory and Academic Division of Clinical Rheumatology, Department of Internal Medicine, IRCCS Polyclinic Hospital San Martino, University of Genoa, Genoa, Italy; ${ }^{2}$ Department of Rheumatology, Ghent University Hospital, Ghent, Belgium; ${ }^{3}$ Department of Internal Medicine, Ghent University, Ghent, Belgium; ${ }^{4}$ Unit for Molecular Immunology and Inflammation, VIB Inflammation Research Center (IRC), Ghent, Belgium

Background: The nailfold videocapillaroscopy (NVC) analysis allows microvascular detection of possible markers of severity and progression in systemic sclerosis (SSc), as reduced capillary number, which has been associated with a high risk of developing disease complications (i.e. digital ulcers over time) (1-2). A recent study validated an automated counting system (AUTOCAPI) for the absolute nailfold capillary number in SSc patients by analysing the capillaroscopic images (3).

Objectives: To evaluate the performance of this automated software for absolute nailfold capillary number counting, in SSc patients with different NVC patterns of microangiopathy.

Methods: 183 random SSc patients collected at both Genova and Ghent Divisions of Rheumatology were enrolled (LeRoy 2001 or ACR 2013 criteria, mean age $55 \pm 13$ year, mean disease duration $5.5 \pm 6.8$ years) and classified by NVC in the following patterns: 28 "not specific", 37 "early", 89 "active", 29 "late"). 8 fingers for each patient were analysed, counting the number of nailfold capillaries manually and by the AUTOCAPI software (DS Medica, Italy) along a millimetre in each finger image. The mean capillary number value of the eight finger images was calculated. The software reliability was assessed through calculation of the intraclass correlation coefficient (ICC) between automatic and manual counting

Results: The mean number of capillaries assessed by manual vs automatic counting was as follows: $5.23 \pm 1.7$ vs $5.47 \pm 1.3$ in the total group of SSc patients, $5.91 \pm 1.2$ vs $6.87 \pm 1.2$ in the "not specific", $7.23 \pm 1.4$ vs 5.67 \pm 1.1 in the "early", $4.67 \pm 1.1$ vs $5.16 \pm 1.2$ in the "active" and $3.72 \pm 1.5$ vs $4.85 \pm 1.1$ in the "late" pattern of microangiopathy. The higher standard deviation observed for automatic counting was 1.23 in the "not specific" group. The following ICC's were obtained respectively for total patients, "not specific", "early", "active", and "late" NVC patterns: 0.53, 0.51, 0.48 , 0.50 and 0.66 . The mean values for the manual versus automatic capillary counting assessed by the two centres in all SSc patients were respectively: $5.92 \pm 1.8$ and $5.02 \pm 1.1$ for Genova centre, and $4.71 \pm 1.5$ and $5.83 \pm 1.4$ for Ghent centre. The automatic counting confirmed that capillary number progressively reduces from "early" to "active" to "late" NVC pattern of microangiopathy in SSc.

Conclusion: This study reports for the first time the good reliability of AUTOCAPI software in nailfold capillary number counting in SSc patients with different patterns of microangiopathy. The use of automated counting software need to standardize nailfold capillary assessment among different Rheumatological centres.

\section{REFERENCES}

[1] Smith V, et al. J Rheumatol. 2013:40:2023-8. 2. Cutolo M, et al. Arthritis Rheumatol. 2016;68:2527-39. 3. Cutolo M, et al. Microcirculation. 2018;25: e12447.

Disclosure of Interests: None declared

DOI: 10.1136/annrheumdis-2019-eular.6781

\section{AB1134 IMPROVING THE STANDARDISATION OF DIAGNOSTIC LABORATORY TESTS FOR RF AND ACPA THROUGH THE ESTABLISHMENT OF A NEW INTERNATIONAL REFERENCE MATERIAL}

Luisa de Jesus Saraiva ${ }^{1}$, Peter Rigsby ${ }^{2}$, Lucy Studholme ${ }^{1} .{ }^{1}$ National Institute for Biological Standards and Control, Biotherapeutics, Potters Bar, United Kingdom; ${ }^{2}$ National Institute for Biological Standards and Control, Biostatistics, Potters Bar, United Kingdom

Background: Rheumatoid arthritis is a debilitating chronic inflammatory disease. Its laboratory diagnosis relies mainly on testing for the presence of two groups of autoantibodies in patient's serum: rheumatoid factor (RF) and anti-citrullinated protein antibodies (ACPA). Most RF assays are standardised against W1066, the WHO international standard rheumatoid arthritis serum established in 1968, or 64/002, the British Standard rheumatoid arthritis serum, which is the same material. Stocks of W1066 are becoming exhausted which triggered a decision by NIBSC and WHO to prepare a new standard preparation intended to replace W1066.

Objectives: Primarily, we have a responsibility to ensure the continuity of W1066 as a calibrator in RF agglutination-based assays. However, science has progressed and ACPA are now recognised as equally important to RF in the diagnosis of rheumatoid arthritis which is reflected in the ACR/EULAR 2010 classification guidelines. Therefore, we intend to assign the new standard values in international units to both RF and ACPA. In addition, some assays look at isotype-specific RF, so we will assess whether we can establish nominal values for the most common isotypes, IgM-RF and IgA-RF.

Methods: A pool of rheumatoid arthritis serum was prepared and dispensed into ampoules, lyophilised and sealed under an inert nitrogen atmosphere. An international collaborative study was organised with expert laboratories, including commercial kit manufacturers and medical 
laboratories, to assess the suitability of the new candidate standard and calibrate it against W1066.

Results: The results of the collaborative study will be used to propose a unitage to the new candidate standard in RF and ACPA for consideration at the WHO Expert Committee for Biological Standardisation.

Conclusion: Standardisation of diagnostic laboratory tests involves the development of a physical sample for each analyte. The use of these international reference standards as primary calibrants promotes the harmonisation of test results between different assays. Because the assays use a similar scale, their results are easily understood, which ultimately increases the impact of published research and clinical findings on our knowledge of the disease.

\section{REFERENCES}

[1] WHO Expert Committee on Biological Standardization, sixty-seventh report. Geneva: World Health Organization; 2017 (WHO Technical Report Series; no. 1004). 2017

Disclosure of Interests: None declared DOI: 10.1136/annrheumdis-2019-eular.4068

\section{AB1135 THE MANAGEMENT OF INTERSTITIAL LUNG DISEASES: THE IMPORTANCE OF THE RHEUMATOLOGIC EXPERTISE IN MULTIDISCIPLINARY MEETINGS}

Enrico De Lorenzis ${ }^{1}$, Gerlando Natalello ${ }^{1}$, Lucrezia Verardi ${ }^{1}$, Silvia Laura Bosello ${ }^{2}$, Elisa Gremese ${ }^{1,2} .{ }^{1}$ Catholic University of the Sacred Heart, Institute of

Rheumatology, Rome, Italy, ${ }^{2}$ Fondazione Policlinico Universitario A. Gemelli IRCCS, Department of Rheumatology, Rome, Italy

Background: Multidisciplinary Team (MDT) meetings are the current "gold standard" in interstitial lung disease (ILD) diagnosis. Requisite participants are respiratory physicians, radiologists and pathologists. A rheumatologist physician is not routinary involved in MDT even if up to $20 \%$ of ILD are related to connective tissue disease, rheumatoid arthritis or systemic vasculitis.

Objectives: The aim of this study is to evaluate the prevalence and predictors of systemic rheumatological diseases in a cohort of patients with ILD, evaluated by a rheumatology specialist on the advice of MDT in a university hospital.

Methods: Thirty-two patients with ILD, evaluated at dedicated MDT were referred to a rheumatologist in 2018, usually for autoantibodies positivity or clinical history suspected for a rheumatological disorder. Rheumatologic evaluation included physical examination, routinary blood and urine tests, serum levels of $\mathrm{C} 3$ and C4, ANA, Rheumatoid Factor (RF), ANCAs, antiSm, anti-RNP, anti-Ro/SSA, anti-La/SSB, anti-Sm, anti-Jo1, anti-dsDNA and anti-CCP antibodies. Family history of autoimmune diseases, presence of rheumatologic red flags (Raynaud's phenomenon, photosensitivity, inflammatory skin manifestations, sicca syndrome, recurrent fever, inflammatory arthralgias, paresthesias, oral or genital aphthosis, thrombosis and recurrent miscarriages), respiratory symptoms and pulmonary function test were also evaluated. When indicated, capillaroscopy, joint imaging and salivary gland biopsy were performed.

Results: Twenty-one patients $(65.6 \%)$ were female. At the time of rheumatological evaluation, the patients had a mean age of $64.4 \pm 12.6$ years with a mean period of $21.1 \pm 39.6$ months since the first identification of ILD on a CT scan. Twenty-five (78.1\%) and 18 (56.3\%) patients respectively complained exertional dyspnea and cough, 6 patients $(18.8 \%)$ were on oxygen therapy and $13(40.6 \%)$ had a $\mathrm{FVC}<80 \%$. The pattern on CT scan suggested a UIP, NSIP and OP respectively in 18 (56.3\%), 6 $(18.8 \%)$ and $2(6.3 \%)$ patients. Ten patients $(31.3 \%)$ were diagnosed with a defined rheumatological condition (4 with Sjogren Syndrome, 3 with scleroderma, 1 with Rheumatoid Arthritis, 1 with Mixed Connective Tissue Disease and 1 with Granulomatosis with Polyangiitis); 7 (21.9\%) were diagnosed with Interstitial Pneumonia with autoimmune feature (IPAF). A rheumatological diagnosis was not statistically associated with any specific rheumatologic red flag, routine laboratory abnormalities or family history of systemic autoimmune disease. Three out of 13 capillaroscopies and 3 out of 9 salivary glands biopsies performed had diagnostic findings. The only immunologic abnormalities associated to a rheumatologic diagnosis were RF positivity $(20.0 \%$ vs $54.5 \%, \quad p=0.024)$ and p-ANCA positivity $(10.0 \% v s 40.9 \%, \quad p=0.042)$, both with low specificity (respectively $15.0 \%$ and $5.0 \%$ ) and sensitivity (respectively $55.6 \%$ and $42.9 .0 \%$ ). After diagnosis, 11 patients $(34.4 \%)$ started immunosuppressive therapy and 7 $(21.9 \%)$ started antifibrotic therapy.

Conclusion: Single clinical or laboratory abnormalities are not strongly associated to a rheumatological diagnosis in patients with ILD, suggesting that only a comprehensive rheumatological evaluation allows correct classification of the disease associated with ILD and is mandatory to make or exclude a diagnosis. In this study, we evaluated only preselected patients by ILD-MDT, but the results indirectly suggest that direct participation of rheumatologist to MDT is advisable to increase accuracy and reduce delay in diagnosis and treatment.

Disclosure of Interests:: Enrico De Lorenzis: None declared, Gerlando Natalello: None declared, Lucrezia Verardi: None declared, Silvia Laura Bosello: None declared, Elisa Gremese Consultant for: AbbVie, BMS, Celgene, Janssen, Lilly, MSD, Novartis, Sanofi, UCB, Roche, and Pfizer Speakers bureau: BMS, Speakers bureau: Roche, Speakers bureau: AbbVie, BMS, Celgene, Janssen, Lilly, MSD, Novartis, Sanofi, UCB, Roche, and Pfizer

DOI: 10.1136/annrheumdis-2019-eular.6364

\section{AB1136 CD26: A POTENTIAL NOVEL HISTOLOGICAL MARKER OF IDIOPATHIC INFLAMMATORY MYOPATHIES}

$\underline{\text { Rebecca De Lorenzo }}^{1}$, Clara Sciorati ${ }^{1}$, Antonella Monno ${ }^{1}$, Francesco Bonomi ${ }^{2}$, Silvia Cavalli ${ }^{2}$, Antonio Francesco DI Naro ${ }^{3}$, Stefano Previtali ${ }^{4}$, Patrizia RovereQuerini ${ }^{1}$. 1 San Raffaele Scientific Institute, Laboratory of Innate Immunity and Tissue Remodelling, Milan, Italy; ${ }^{2}$ Vita-Salute San Raffaele University, Milan, Italy, ${ }^{3}$ Adienne Pharma and Biotech, Lugano, Switzerland; ${ }^{4}$ San Raffaele Scientific Institute, Experimental Neurology Institute (INSPE), Milan, Italy

Background: Idiopathic inflammatory myopathies (IIM) are a heterogeneous group of acquired skeletal muscle disorders including polymyositis (PM), dermatomyositis (DM), inclusion body myositis (IBM) and immunemediated necrotizing myopathy (IMNM), characterized by immune-mediated muscle damage ${ }^{1}$. Activated $\mathrm{T}$ cells are the predominant inflammatory infiltrates in muscle biopsies of PM and DM patients and the lack of $\mathrm{T}$ regulatory cells $\left(T_{\text {regs }}\right)$ has been implicated in the persistence of muscle damage. CD26 is an intrinsic membrane glycoprotein and a serine exopeptidase involved in the activation of $T$ lymphocytes and amplification of inflammatory cytokines production. The enzymatically active form of CD26 is selectively expressed by activated $\mathrm{T}$ cells and has been described as a negative selection marker for human $\mathrm{T}_{\text {regs }}{ }^{2-5}$.

Objectives: The aims of this study were to evaluate the expression of CD26 in muscle biopsies of IIM patients and to correlate it with patients' clinical and histological features.

Methods: Immunofluorescence was used to evaluate CD26 expression and co-localization with CD3 and CD31, markers of T cells and endothelial cells respectively, in muscle biopsies of $6 \mathrm{DM}, 6 \mathrm{PM}, 3 \mathrm{IBM}$ and 3 IMNM patients and of 6 healthy controls.

Results: We found that CD26 is preferentially expressed in muscle biopsies of IIM patients with respect to controls and that its level of expression is higher in DM patients. In muscle biopsies of IIM patients, CD26 is distributed not only in the extracellular matrix surrounding myofibers and infiltrating leukocytes, but also at the level of $\mathrm{T}$ cell membranes and endothelial cells. Specifically, CD26 co-localization with CD31 is more prominent in DM muscle biopsies. We could not find any association between vessel morphology in terms of size and shape and CD26 endothelial expression, suggesting that CD26 is expressed at the perivascular level independently of the degree of vessel dysfunction. With regard to clinical features, we found that CD26 is more expressed in patients presenting the typical DM rash. Moreover, CD26 expression was found not to be significantly associated with the degree of muscle weakness nor with the presence of interstitial lung disease, dysphagia, myalgias or mechanic's hands. As for histological data, higher levels of CD26 expression were found in biopsies with perivascular inflammatory infiltrates, especially $\mathrm{T}$ lymphocytes and macrophages.

Conclusion: Our data suggest that CD26 may represent a suitable marker for the diagnosis of IIM and a potential novel target for selective immune-therapies.

\section{REFERENCES}

[1] Dalakas, M. C. (2015). "Inflammatory Muscle Diseases." N Engl J Med 373(4): 393-394.

[2] Goebels N, Michaelis D, Engelhardt M, et al. Differential expression of perforin in muscle-infiltrating $\mathrm{T}$ cells in polymyositis and dermatomyositis. $J$ Clin Invest 1996;97:2905-10.

[3] Waumans Y, Baerts L, Kehoe K, Lambeir A M, De Meester I. The dipeptidyl peptidase family, prolyl oligopeptidase, and prolyl carboxypeptidase in the immune system and inflammatory disease, including atherosclerosis. Front Immunol 2015; 6:387. 